\title{
Avaliação do acesso a periódicos eletrônicos na web pela análise do arquivo de log de acesso*
}

\author{
Guilherme Ataíde Dias \\ Bacharel em Ciência da Computação - Universidade Federal da Paraíba \\ - 1990. Mestre em Administração - Central Connecticut State University, \\ USA -1996. Doutorando em Ciência da Informação - Universidade de \\ São Paulo, ECA/USP. \\ E-mail: guilhermeataide@aol.com
}

\section{Resumo}

Este artigo apresenta uma abordagem sobre a avaliação do acesso a periódicos eletrônicos disponibilizados na World Wide Web por meio da análise do arquivo de log de acesso. $O$ arquivo de log de acesso da revista Informação \& Sociedade: Estudos é processado e apresentado como um exemplo de aplicação do uso de uma ferramenta automatizada de análise para arquivo de log de acesso. As características inerentes à análise do arquivo de log de acesso são apresentadas e discutidas.

\section{Palavras-chave}

Periódicos eletrônicos; Avaliação de acesso; Arquivo de log de acesso.

Evaluating the access of electronic periodicals at the Web through the analysis of the access log file

\section{Abstract \\ This article presents an approach for the evaluation of the access to electronic journals made available in the World Wide Web through the analysis of the access log file. The access log file of the journal Informação \& Sociedade: Estudos is processed and presented as an example of the use of an automated tool for log file analysis. The inherent features on the analysis of the access log file are presented and discussed.}

Keywords

Electronic periodicals; Access evaluation; Access log file.

\section{INTRODUÇÃO}

O processo de disponibilização de um periódico eletrônico na World Wide Web é um empreendimento composto de várias etapas. A partir do momento em que estas etapas estejam completas, torna-se necessário avaliar de alguma maneira o acesso ao respectivo periódico eletrônico pelos seus usuários. Pode-se medir, por exemplo, dentre uma variedade de opções, os seguintes itens: o acesso à página de entrada (home page), acessos aos resumos dos artigos, acesso ao texto completo dos artigos (download de artigos). A importância em estudar o acesso a periódicos eletrônicos traduz-se de várias maneiras: é importante ter conhecimento dos conteúdos acessados pelos usuários como forma de identificar as suas necessidades e atendêlos de forma adequada; no caso de uma biblioteca disponibilizar o acesso a periódicos eletrônicos pagos, é fundamental ter uma estatística de acessos a estes periódicos como forma de justificar o investimento feito em assinaturas.

Os dados compilados neste artigo contemplam o acesso ao texto completo dos artigos disponibilizados no periódico eletrônico Informação $\mathcal{E}$ Sociedade: Estudos, uma publicação semestral do Curso de Mestrado em Ciência da Informação da Universidade Federal da Paraíba (CMCI/UFPB). O periódico em questão passou a ter uma versão eletrônica a partir do primeiro semestre de 2000 e pode ser acessado através da URL http:// www.informacaoesociedade.ufpb.br. A versão impressa de forma tradicional em papel continua sendo editada.

\section{DADOS SOBRE USO DOS PERIÓDICOS ELETRÔNICOS}

Por ser uma atividade relativamente nova, a análise do acesso a periódicos eletrônicos apresenta alguns pontos passíveis de análise e discussão, pois estes pontos de uma forma geral precisam ser compreendidos e normatizados. Segundo Luther (2000), um problema fundamental sobre o qual não se chegou ainda a um entendimento seria o de

\footnotetext{
* Este artigo faz parte da tese de doutorado em ciência da informação do autor, que tem como orientadora Dinah Población.
} 
como gerar dados de modo que os mesmos pudessem ser comparadose utilizados.

Tomando-se como exemplo os periódicos científicos eletrônicos brasileiros na área da ciência da informação, apenas o periódico C iência da I nformação, hospedado no SciELO , apresenta de forma aberta para a comunidade de usuários a opção de consultar estatísticas. 0 s relatórios de utilização disponíveis são os deacessos da revista, acessos aos fascículos e acessos aos artigos. D e acordo com 0 sugerido em Luther (2000), pode-se inferir que seriainteressante estabelecer uma padronização na geração dos dados associados aos diferentes periódicos eletrônicos. Q ualquer tentativa de comparação do acesso aos sites destas revistas ficaria comprometida sem uma prévia padronização dos dados estatísticos a serem gerados. U ma maneira de contornar a inexistência de uma padronização de dados a serem processados e comparados pode ser feita com autilização dos arquivos de log gerados pelos servidores web. Estes arquivos de log podem eventualmente apresentar diferenças no formato em virtude da diversidade de servidores web existentes, contudo é possível configurar os diferentes servidores web paragerar um arquivo delog de acesso deacordo com uma especificação única e amplamente conhecida. Esta especificação é o C ommon L ogfileF ormat (C LF) tal qual detalhado pelo World WideWeb Consortium (W3C). 0 World Wide Web C onsortium pode ser acessado através daU RL http://www.w3.org.

N estetrabalho, considera-sequeum logéum registro com informações relativas à ocorrência de determinados eventos. A seguir, são apresentados dois exemplos práticos do que vem a ser um log:

1. sempreque determinado artigo disponibilizado em um periódico eletrônico éconsultado (evento), informações relativas a esta consulta (log) são armazenadas em um arquivo;

2. em uma biblioteca, quando um usuário solicita o empréstimo deum livro (evento), as informações relativas a esteempréstimo são armazenadas em linhas deumaficha (log) e posteriormente armazenadas em um arquivo.

A seguir apresenta-se, de forma detalhada, como cada entrada no arquivo de log deacessos do servidor web deve estar estruturada de acordo com o C ommon L ogfileF ormat:
MáquinaR emota rfc931 U suárioAutenticado [ data] "requisição" status bytes

Cada campo do C ommon Logfile F ormat armazena as seguintes informações:

MáquinaR emota: o nomedamáquinaremota ou o endereço IP no caso de o nome da máquina não estar disponível;

rfc931: nome do usuário remoto, se a informação não estiver disponível, um sinal demenos ( - ) será colocado no campo;

U suárioAutenticado: no caso de o documento requisitado ser protegido por uma senha de acesso, então este campo conteráo nome do usuário autenticado (L aurie,1999); se a informação não estiver disponível, um sinal de menos ( - ) será colocado no campo;

[ data] : data ehora de requisição do documento;

"requisição": a linha da requisição exatamente como solicitada pelo cliente;

status: código de três dígitos retornado para o cliente indicando o status da requisição;

bytes: o tamanho em bytes do conteúdo transferido.

A linha apresentadaa seguir (figura 1), extraídado arquivo de log do servidor web A pache, onde está hospedado 0 periódico eletrônico I nformação \& Sociedade: E studos, permite-nos apresentar na prática a forma de um arquivo deloggerado deacordo com o C ommon L ogfileF ormat. 


\section{ACESSO A PERIÓDICOS ELETRÔNICOS EMSITES DA WEB}

A partir do exposto, serão mostrados alguns tipos de relatórios que podem ser obtidos no acesso desitesda web mediante análise dos arquivos de log de acesso. Em um primeiro momento, os arquivos de log de acessos podem apresentar-se como a solução ideal para a análise do acesso a si tes da w eb. C ontudo, é importante mencionar que os arquivos de log de acesso nos oferecem recursos para que sejam realizadas análises apenas de cunho estritamente quantitativo, facilitando a identificação de questões relativas a "o quê", "quando" e "por quem".

D e acordo com H aigh (1998), os dados contidos em um arquivo de logde acessos podem ser processados para gerar relatórios, tais como:

- total de arquivos ekbytes servidos com sucesso;

- número distinto de endereços IP servidos e número de requisições associadas a cada endereço;

- número de requisições feitas por sufixos de domínios;

- número de requisições para arquivos específicos ou diretórios;

- totalizações emédias por períodos específicos de tempo (horas, dias, semanas, meses, anos);

- U RLs visitadas anteriormentepelo usuário (informação indisponível no caso de utilização do C ommom Logfile Format).

Paraobterem-serelatórios extraídos a partir de um arquivo de log de acesso, é recomendável fazer-se uso de uma ferramenta automatizada adequada para tal fim. É possível, contudo, anal isar-se o conteúdo de um arquivo de log de acesso de forma manual, porém tal procedimento não é recomendável, pois o tamanho do referido arquivo freqüentemente possui milhares de linhas, se for considerado queo sitehospedeiro do periódico eletrônico estudado seja mediamente visitado. U ma listagem contendo diversas ferramentas automatizadas para a análisedelogdeacesso podeser encontradanaU RL http:/ /www.w3.org/WCA/loganalysis-tools.html.

A figura 2 e as tabelas 1 e 2, a seguir, são exemplos de relatórios obtidos a partir da anál ise de um arquivo de log deacesso. 0 arquivo delog de acesso utilizado foi obtido do si teda web onde estáhospedado o periódico eletrônico I nformação \& Sociedade: E studos. 0 gráfico e as tabelas mostram o número de vezes que os arquivos associados a artigos mais acessados do periódico foram "baixados" (downloaded) no período entre $1^{\circ}$ demarço de 2000 e $1^{\circ}$ de novembro de 2000. A ferramenta utilizada para auxiliar a geração dos relatórios apresentados foi o softwareWebtrends L og Analyzer. $M$ ais informações sobre esta ferramenta podem ser encontradas pela URL http:// www.webtrends.com.

\section{PROBLEMAS NA UTILIZAÇÃO DE DADOS EM ARQUIVOS DE LOG DE ACESSO}

Serão analisados agora, de maneira detalhada, alguns problemas associados ao uso dos dados contidos nos arquivos de log de acesso. Paraisso, énecessário que sejam apresentadas al gumas definições. O stermos "hit" e "sessão de usuário (user session)" serão explicados:

- H it: todatroca de dados realizada entre um clienteeum servidor web. Exemplo: um usuário solicita, através de seu navegador, (browser) uma página H TM L (.html). N este caso, tem-se um hit. Supondo que este usuário acesse uma outra página com três imagens associadas à mesma, temse então quatro hits, um hit para a página H TM L e mais três para os arquivos de imagem associados. 
- Sessão de usuário (user session): uma sessão de usuário pode ser definida por meio da delimitação de um período de tempo em que ocorrem solicitações ao servidor web provenientes de um mesmo endereço IP. Uma sessão é considerada encerrada após determinado período de inatividade, por exemplo, de 30 minutos. As ferramentas usadas para analisar arquivos de $\log$ de acesso utilizam variações desta seqüência apresentada para determinar uma sessão de usuário.

Com certa freqüência, encontrase publicado, nos periódicos especializados, o número de hits que determinado site obteve em um período de tempo. Esta informação é publicada com o intuito de quantificar o número de acessos a determinado site. A medição do número de acessos de um site, baseada no número de hits, não fornece um indicador confiável, pois, de acordo com o exposto na definição de hit, uma página consultada uma só vez pode gerar mais hits do que uma página que seja consultada várias vezes, mas que gere uma quantidade menor de hits. Conseqüentemente, não se recomenda utilizar o número de hits como medida para analisar o acesso a periódicos eletrônicos disponibilizados em sites da web.

$\mathrm{O}$ processo de contagem e identificação de sessões de usuários não é preciso, pois não se pode associar com total segurança um endereço IP a um único usuário. No caso de o usuário estar utilizando uma estação de trabalho com endereço IP estático, esta estação, mesmo que esteja sendo utilizada por uma dezena de usuários diferentes, vai apresentar sempre o mesmo endereço IP,
TABELA 1

Revista Informação $\mathcal{E}$ Sociedade: Estudos - arquivos mais baixados entre 1을 de março de 2000 a $1^{\circ}$ de novembro de 2000

\begin{tabular}{|c|c|c|c|c|}
\hline \multicolumn{5}{|c|}{ Arquivos mais baixados } \\
\hline & Arquivo & $\begin{array}{l}\text { Número de } \\
\text { Downloads }\end{array}$ & $\begin{array}{l}\text { \% Sobre o Número } \\
\text { de Downloads }\end{array}$ & Visitas \\
\hline 1 & 929914.pdt & 652 & $5.43 \%$ & 354 \\
\hline 2 & 929902.pdt & 557 & $4.63 \%$ & 347 \\
\hline 3 & 919903.pdt & 695 & $5.78 \%$ & 342 \\
\hline 4 & 929901.pdf & 609 & $5.07 \%$ & 333 \\
\hline 5 & $929903 \mathrm{pdf}$ & 496 & $4.13 \%$ & 314 \\
\hline 6 & 919901.pdf & 442 & $3.68 \%$ & 307 \\
\hline 7 & 919905.pdf & 495 & $4.12 \%$ & 288 \\
\hline 8 & 919908.pdt & 470 & $3.91 \%$ & 283 \\
\hline 9 & 929905.pdt & 431 & $3.58 \%$ & 280 \\
\hline 10 & 929908.pdt & 363 & $3.02 \%$ & 270 \\
\hline 11 & 929915.pdt & 314 & $2.61 \%$ & 249 \\
\hline 12 & 929907.pdt & 282 & $2.34 \%$ & 248 \\
\hline 13 & 929916.pdf & 283 & $2.35 \%$ & 244 \\
\hline 14 & 929922. pdf & 288 & $2.39 \%$ & 243 \\
\hline 15 & 919909.pdf & 291 & $2.42 \%$ & 242 \\
\hline 16 & 929904.pdt & 282 & $2.34 \%$ & 241 \\
\hline 17 & 929911.pdt & 302 & $2.51 \%$ & 239 \\
\hline 18 & 929906.pdt & 277 & $2.3 \%$ & 237 \\
\hline 19 & 919904.pdf & 292 & $2.43 \%$ & 236 \\
\hline \multirow{2}{*}{\multicolumn{2}{|c|}{$\begin{array}{l}20 \\
\text { Totais }\end{array}$}} & 265 & $2.2 \%$ & 235 \\
\hline & & 8,086 & $67.34 \%$ & N/A \\
\hline
\end{tabular}

TABELA 2

Revista Informação $\mathfrak{G}$ Sociedade: Estudos-títulos dos artigos mais baixados entre 1ํ de março de 2000 a $1^{\circ}$ de novembro de 2000

\begin{tabular}{|c|c|c|c|}
\hline \multicolumn{4}{|c|}{ Titulos male balixadoen } \\
\hline & Arquitwo & Nome do Artigo & Nome de Autor \\
\hline$T$ & 929914.pd & $\begin{array}{l}\text { OS DESTINOS DA CIENCIA DA INFCAMACAOA antre o cristal } \\
\text { a chama }\end{array}$ & Aldo de Abuquerquo Barrebo \\
\hline$\overline{2}$ & 829002 pd & 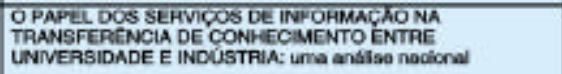 & Fathia Porteta Cysere \\
\hline 3 & 919903 peth & $\begin{array}{l}\text { CONHECIMENTO PAFA O DESENVOLVMENTO! RofexDos } \\
\text { para o profiasional da informagăo }\end{array}$ & $\begin{array}{l}\text { Varia Maria Rodrigues } \\
\text { Hormoe do Araclo } \\
\text { lea Meria Fretre }\end{array}$ \\
\hline 4 & B29001.pd & 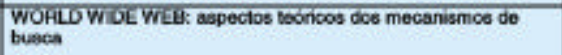 & Fomanda Nahuz \\
\hline$\frac{5}{5}$ & 929000 pall & 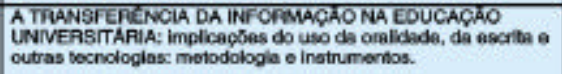 & Hentietle Fecteira Gomes \\
\hline 6 & 918901.pdI & 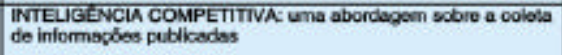 & $\begin{array}{l}\text { Marifa Domiani Coeta } \\
\text { Iranise Alves da Sitha }\end{array}$ \\
\hline 7 & 918006.pd & 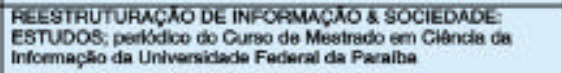 & $\begin{array}{l}\text { Joana Cool Pibeiro Garcia } \\
\text { Maria das Grapas Targho }\end{array}$ \\
\hline 8 & 919308.pdI & INFOAMMACAO PARA MUDANCA \$OCIAL. & 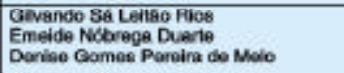 \\
\hline 9 & 929906 pdi & 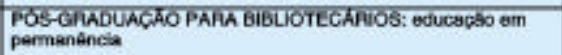 & Jemients Marques do Clveira \\
\hline 10 & 8298cepell & $\begin{array}{l}\text { PRATICAS DE INFCOMAGAO NO ENSENO DE } \\
\text { BIBLIOTECONOMIA }\end{array}$ & Maria Niza Barboesa Roses \\
\hline 71 & 929015 par & $\begin{array}{l}\text { A POCINCA GOVERINAMENTAL PAPA A POS-GAADUAGAO } \\
\text { EM CIENCIA DA INFORMACAO NO BRASIL. }\end{array}$ & Johana W. Gmit \\
\hline 12 & 829907 pdi & $\begin{array}{l}\text { INEFMACAO E VIOLACAO DA CIDADANIIA NOS } \\
\text { PRESIDUOS }\end{array}$ & Josinddo José Femisndes Malsquías \\
\hline 13 & B289iapdi & GLCOALZACAO EMETODO IMPAESSIONSTA & Marla Aurdiadora de Carvel ho \\
\hline 14 & 020022 pdt & 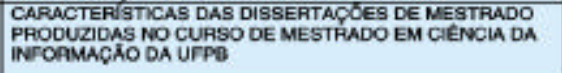 & Mariono do Cliwuirs \\
\hline 15 & 818900.pd & $\begin{array}{l}\text { LETHARK EM SALA DE AULA: avatames do doeeja ou } \\
\text { resalcamento? }\end{array}$ & Mrian do Albuquerque Aquino \\
\hline 16 & $829904 \mathrm{pd}$ & 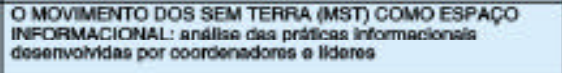 & Hodida Coutinko Barbesa \\
\hline 17 & 929011.pet & 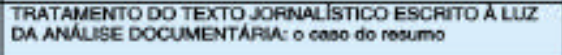 & Ridedi Modeiros \\
\hline 18 & 929906.pdt & 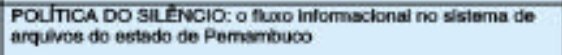 & Josesmar Henrique de Melo \\
\hline 19 & $918904 \mathrm{pd}$ & 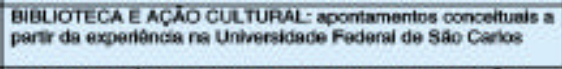 & 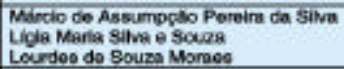 \\
\hline 20 & 929920 pd & $\begin{array}{l}\text { O BIEUOTECAAFIO E O MEFCADO DA DOCUMENTACAO } \\
\text { POPULAR: CPDCS }\end{array}$ & Lectana Moreira Carvaliho \\
\hline
\end{tabular}


Avaliação do acesso a periódicos eletrônicos disponibilizados na World WideWeb através da análise do arquivo de log de acesso

gerando, destaforma, só uma sessão deusuário. D emaneira contrária, no caso de se utilizar uma estação que trabalha com endereços IP dinâmicos, tem-se umavariedade de sessões de usuários, quando, na realidade, existe a possibilidade de essas sessões estarem associadas aum só usuário. Portanto, contar e identificar sessões de usuários fornece, apenas, uma estatística aproximada do número deusuários distintos e do número de vezes que os respectivos acessaram o site da web, hospedeiro do periódico eletrônico.

U m evento quetambém pode afetar o processo de determinar uma sessão, bem como aumentar 0 número de hits no servidor web, seria a visita de um software do tipo robot (espécie de navegador automático) que faz a varredura completa de um site. $D$ a mesma forma que um usuário comum, um robot também tem suas atividades registradas no arquivo de log de acesso. Esse tipo de software está normalmente associado a sites que disponibilizam ferramentas de busca. A I guns softwares para a análise de arquivos de log permitem que seja isolado o uso gerado por robots (H aigh, 1998), contribuindo, portanto, para reduzir a incidência de erro quando da análise dos arquivos de log de acesso.

Para complementar esta explanação sobre os problemas oriundos da utilização de dados armazenados nosarquivos de log de acesso, será explicado o conceito deuma cachede dados. N o jargão da ciência da computação, a cache é entendida como umaárea onde dados são armazenados de forma temporária. A função primordial de uma cachede dadosépermitir que usuários tenham acesso à informação de maneira otimizada.

A utilização da cache de dados permitequeas informações solicitadas pel os usuários sejam recuperadas de maneira mais veloz. M as, em compensação, pode reduzir a significância das informações contidas nos arquivos de log de acesso, pois um usuário pode recuperar um determinado artigo e esta ação pode não ficar registrada no arquivo delog de acesso do servidor web. $\mathrm{N}$ o caso deum usuário requisitar um artigo de periódico através de uma $U \mathrm{RL}$ digitada em seu browser, pode-se verificar algumas situações distintas*:

1. A página associada ao artigo de periódico já está armazenada na cache local do próprio browser. Todo o processo é realizado na própria estação de trabal ho do usuário. C onseqüência: 0 arquivo delogdeacesso associado ao servidor web, ao qual o artigo foi requisitado, nunca vai registrar tal solicitação, pois a requisição já foi satisfeita pela própria cachelocal do browser (situação representada nafigura 3);

2. A página associada ao artigo de periódico não está armazenada na cache local do próprio browser, mas na cache do provedor de acesso à internet do usuário, em virtude de outro usuário já ter requisitado o mesmo artigo de periódico, anteriormente. Conseqüência: 0 arquivo de log de dados associado ao servidor web, ao qual o artigo foi requisitado, nunca vai registrar tal solicitação, pois a requisição já foi satisfeita pela cache do provedor de acesso à I nternet do usuário ( situação representada na figura 4);

\footnotetext{
* Situações de caráter ilustrativo. É possível a existência de outras variantes.
} 
3. A página associada ao artigo de periódico não estáarmazenadana cachelocal do próprio browser, nem na cache do provedor de acesso à Internet, mas diretamente no servidor web, associado à U RL digitada pelo usuário no browser. C onseqüência: 0 arquivo de log de acesso associado ao servidor web ao qual 0 artigo foi requisitado vai ter a solicitação registrada (situação representada nafigura 5).

\section{CONCLUSÃO}

A pós estas considerações relativas ao uso dos arquivos de log de dados, torna-se evidente a necessidade de ter-se bastantecuidado sempreque for necessário gerar análises baseadas nestas informações. Parafraseando G oldberg (2001), é possível concluir que atribuir sentido para informações sem nenhum sentido é pior do que não ter nenhuma informação.

U m assunto importante que deve ser considerado diz respeito à escolhade umaferramenta automatizada paraa análise do logde acesso. No momento da escolha de uma ferramenta, é fundamental levantar alguns questionamentos como os seguintes: a ferramenta leva em consideração as linhas do loggeradas por robots? C omo esta ferramenta determina uma sessão deusuário? A correta escolha de uma ferramenta automatizada paraa análise de logédecisiva para ter-se uma idéia próxima da realidade das dinâmicas de acesso a um periódico eletrônico.

Foi visto, no decorrer destetexto, que, mediante análise dos arquivos de log de acesso, não se pode ter um perfil completamente preciso do acesso a periódicos eletrônicos hospedados em sites da web, mas apenas um modelo aproximado do que acontecenarealidade, pois estaéuma abordagem quantitativa que não fornece subsídios para endereçar questões de caráter qualitativo, tais como a opinião dos usuários com relação ao conteúdo do site, satisfação, usabilidade e os motivos queo levaram aacessar o site. U m perfil mais realista, não só deacesso, mastambém de uso, deve ser elaborado em conjunto com outras técnicas tais como entrevistas, preenchimento de questionários egruposfocais.
Relato deExperiência aceito para publicação em 07-09-2001.

\section{REFERÊNCIAS BIBLIOGRÁFICAS}

LAU RIE, Ben, LA U RIE, Peter. A pache: the definitiveguide. 2. ed. [S. I.] : Sebastopol:0 'R eilly, 1999. $369 \mathrm{p}$.

LU TH ER, J udy. Whitepaper on electronic journal usagestatistics. J ournal of Electronic Publishing, v. 6, n. 3, mar. 2000. D isponível em: < http:// www.press.umich.edu/jep/06-03/luther.html> Acesso em : 12 maio 2001.

GO LD BERG , J eff. Why web usage statisticsare (worsethan) meaningless. D isponível em: < http://www.cranfield.ac.uk/docs/stats/>. A cesso em: 23 maio 2001.

H AI G H , Susan, M E G ARITY, J anette. M easuring web site usage: log file analysis. N etwork N otes, n. 57, ago. 1998. D isponível em: < http:// www.nlcbnc.ca/ 9/1/p1-256-e.html. A cesso em: 20 maio 2001. 